\title{
Port machinery and equipment performance analysis system
}

\author{
Zhongfei Kang ${ }^{1, a}$, Xu Guo ${ }^{2, b}$ \\ ${ }^{1,2}$ Number2618, Xingang road 2 , Tanggu district , Tianjin city , China \\ a642456933@qq.com, ${ }^{\mathrm{b}} 18630836305 @ 163 . c 0 m$
}

\begin{abstract}
Keywords: Port machinery,Performance information collection,Wireless transmission,Labview system.
\end{abstract}

\begin{abstract}
With the development of shipping and continuous promotion of energy-saving work, the performance level of port machinery has become an important parameter to measure the comprehensive utilization of energy in all port enterprises. At this stage, the continuous development and improvement of remote monitoring technology, real-time monitoring of the port machinery parameters through the software system,timely detection of problems, can increase the efficiency of enterprises,reduce the impact of failure on production activities and improve the economic efficiency of enterprises. This paper explores the related problems of port machinery information acquisition,introduces the composition of hardware and software of performance analysis system and the main functions of the system,and proposes the direction of expanding and improving.
\end{abstract}

\section{Introduction}

Port machinery and equipment is the basis for the survival and development of the port enterprises.In order to enable the port enterprises to carry out their production normally,it is necessary to ensure that machinery and equipment are often in a good technical condition.Improving the reliability of port machinery and equipment,saving maintenance costs under harsh working conditions and prolonging the service life of equipment effectively are problems often faced by port equipment managers and port equipment maintenance technicians[1,2].

In recent years,port machinery monitoring technology advances by leaps and bounds, the port enterprises are all developing software management systems.However, due to different technologies and actual needs,there is a big difference.System can not fully reflect the operation of equipment.At the present stage, the test of the performance parameters can only connect the test instrument to the distribution room of the mechanical equipment which is in operation, which is not conducive to real-time monitoring, but also has some potential safety hazards.The establishment of port mechanical property acquisition and analysis network system can effectively view and count the real-time status of machinery and equipment and capture the problem through the mode of big data to improve the production efficiency.

The development of real-time on-line monitoring technology in various fields not only can reduce manpower input,but also increase the stability and real-time monitoring, make the collected data more accurate,form the historical records to provide analysis and control. 


\section{Port mechanical performance acquisition system overall design concept}

The system mainly collects all kinds of indexes of port machinery through various sensors[3,4]. After signal processing and data collection procedure,it transmits through wireless network and summarizes and processes the information platform of the terminal, and displays the data in the system software. The main flow chart of the system is as follows.

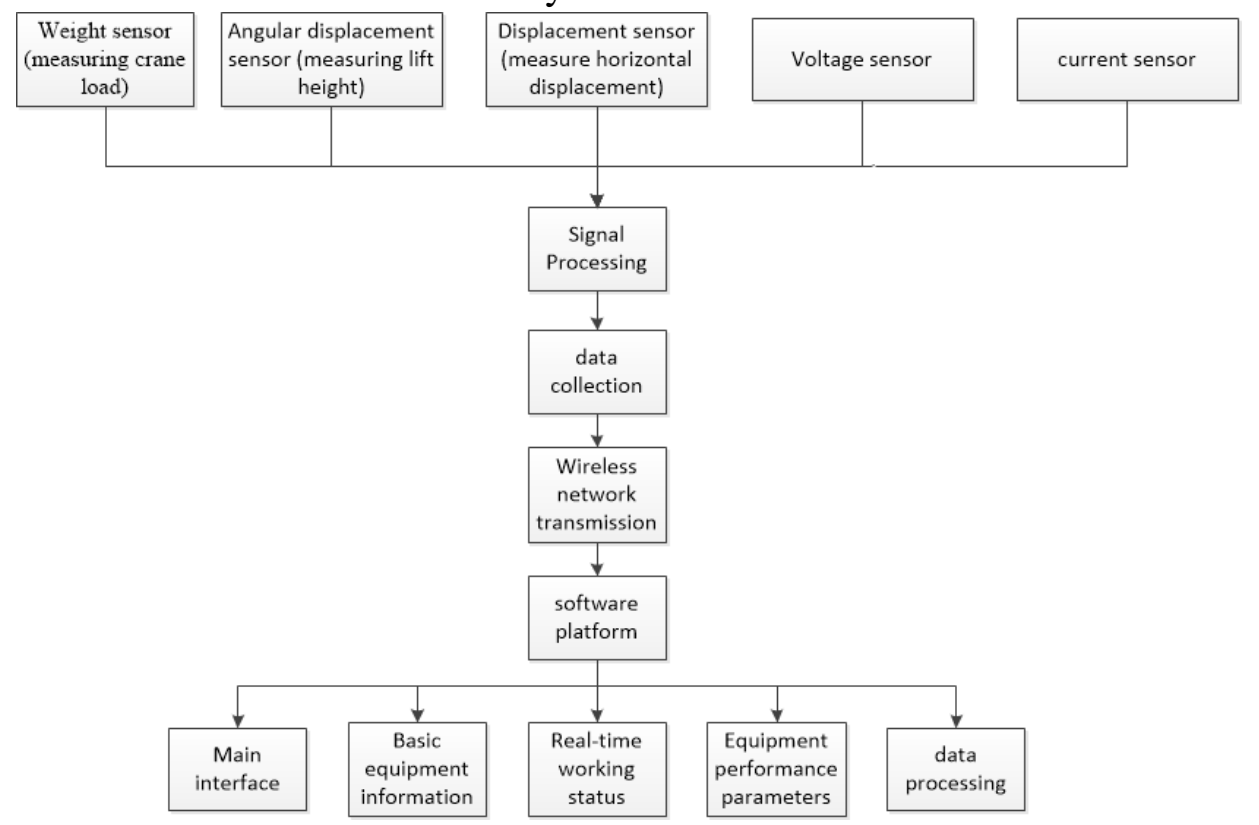

Figure. 1 System flow chart

The main design features of the system is the following

(1) User-friendly interface

In order to make all levels of users can make good use of the system,a friendly interface is essential,click the button on the user interface to complete all operations without the need to understand the operation of the system,everything is running in the background.

(2) The system is highly intelligent

The host computer can analyze the collected data to determine when the value exceeds the pre-set range, which can alert the operator and alert the police,reducing the possibility of danger.

(3) System scalability is good

System hardware and software are modular, structured,standardized, easy system size, function expansion and system interconnection.Major equipment to meet the demolition and reorganization requirements.

(4) The system's fault tolerance is good

System design using star structure,when a remote terminal fails does not affect other remote monitoring point data transmission.

(5) System reliability is good

System has the ability to self-test,hardware and software design also considered the reliability of the problem.

\section{Performance monitoring system hardware and software design}

Hardware design

Data acquisition part 
This project requires a variety of sensors to measure the signal.Including weight sensor,angular displacement sensor,horizontal displacement sensor, voltage sensor, current sensor and so on.

Weight sensor

The weight sensor is actually a device that converts a quality signal into a measurable electrical signal output.For mechanical equipment load measurement.

Angular displacement sensor

Angular displacement sensors, a type of displacement sensor,are patented in a non-contact,patented design that improves long-term reliability compared to other traditional angular displacement meters such as synchronous analyzers and potentiometers.For machinery and equipment to enhance the descent stage height measurement.

Displacement sensor

Displacement sensor, also known as a linear sensor,is a metal-sensitive linear device. The function of the sensor is to convert various measured physical quantities into electrical quantities. Used to measure the distance when the machine is luffing.

Voltage sensor

Voltage sensor is a measured power parameters into DC current,DC voltage and isolated output analog signal or digital signal device.The voltage sensor is used to measure the voltage or current signal with more serious waveform distortion in the power network.It can also measure non-sinusoidal waveforms such as square wave and triangular wave.

\section{Current sensor}

Current sensor is a detection device that can sense the current information,and can detect the sensing of the information, according to a certain law into a certain standard of electrical signals or other forms of information required to meet the output Information transmission,processing,storage, display,recording and control requirements.

Wireless transmission part

4G communication technology has good security, can guarantee the transmission of power quality data security,reliability[5].4G technology uses mimoofdm as a key technology to reduce the impact of frequency-selective fading on bandwidth. $4 \mathrm{G}$ technology has a strong key stream, and a precise encryption algorithm to ensure the safety of data transmission.

In all security system,the mechanism of key agreement and authentication are very important authentication methods.From the beginning of the $3 \mathrm{G}$ communication system, it has been used in the mobile communication system two-way authentication method.The technology can more effectively ensure the user's reliability and authenticity,to prevent malicious attacks.

Therefore, the wireless transmission part of the system uses $4 \mathrm{G}$ communication system to transmit data using 4G wireless communication module based on ME909S-821 for data transmission.It adopts Mini PCIE package to provide the maximum downstream 150Mbps and uplink 50Mbps transmission rate. The embedded TCP/IP protocol stack ,Provide URAT interface, can well realize the interconnection of the network[7].

Software design

In this paper,Labview software is used to make performance monitoring software.LabVIEW (Laboratory Virtual Instrument Engineering Workbench) is a graphical programming language development environment that is widely accepted by industry,academia and research laboratories as a standard Data Acquisition and Instrument Control Software. LabVIEW integrates all the functionality of hardware and data acquisition card communications that meet the GPIB,VXI,RS-232 and RS-485 protocols.It also built a library function to facilitate the application 
of software standards such as TCP/IP, ActiveX.This is a powerful and flexible software.Use it to easily create your own virtual instrument,the graphical interface makes programming and use of the process are lively and interesting[8,9].

The main interface of the system is as follows.

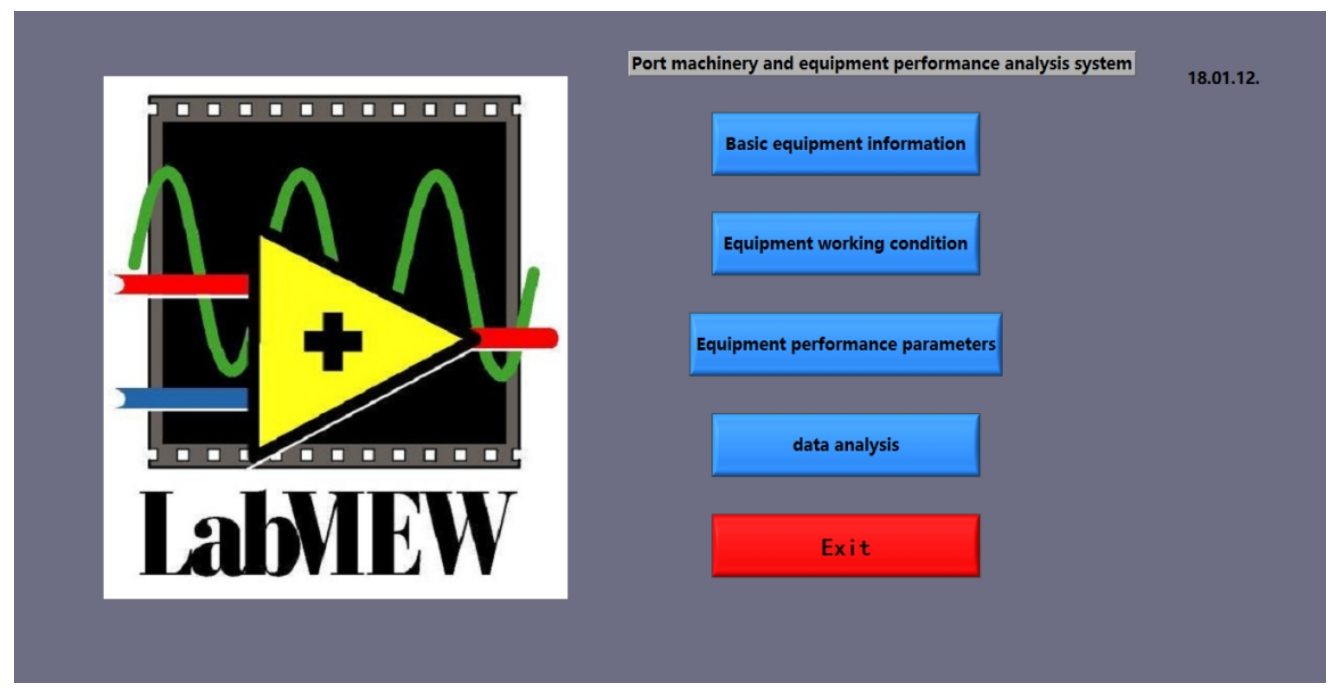

Figure. 2 System main interface

\section{Performance analysis system main function}

Collect device information

Reflect the basic equipment of the information,including the production year,rated power, rated load and so on.Can be reasonably arranged for the use of machinery to maximize efficiency.

Provide equipment real-time status

The working status of the equipment can be checked by software, including lifting,lowering and luffing of quayside and field bridges, including running speed,running speed of trolley and trolley,rotation speed, loading capacity of handling equipment such as forklift and frontal hoist,Master the work of the port machinery.Lifting the weight of the goods.

Check the device performance data

Can view the performance of the device data,such as power,unbalance,power factor,voltage and current parameters, intuitive data control.

Data analysis

The collected data can set quotas for various parameter indicators.If the quota is exceeded,an alarm is triggered,so that problems occurring in the equipment may be promptly checked.

\section{Conclusions}

The use of port enterprises and machinery and equipment is rather complicated.The normal and safe operation of machinery and equipment has become the main standard for the normal operation of enterprises.By collecting the performance information of various machinery and equipment, it is possible to control the usage in real time and find out more clearly during operation problems. The system based on Labview development,user-friendly interface, the underlying development is simple, based on the modular design, is conducive to expanding functionality.Therefore,in actual operation,we can add new modules to meet the different needs according to the differences of equipment in each port enterprise. 


\section{Acknowledgements}

This work was financially supported by the Central-level nonprofit scientific research institutes basic research project special funds (TKS170207).

\section{References}

[1] Zhan Yong, Cheng Haozhong, etc .. Design and development of power quality monitoring and analysis system [J]. East China Electric Power, 2004,10, 10-14.

[2] Xu Lixia. Development of Power Quality Monitoring Technology [J]. China Science and Technology Information, 2012 24, 42-46.

[3] LI Jing, Guo Xia, Talking about the Electric Energy Collection in Power System [J]. Power Technology 2016, 18, 189.

[4] Ho Zhen Shuo 4G mobile communication system security flaws in collaborative communication [J], Communication Design and Application, 2015.6.

[5] Zhu Wei-hua, Suo Da-xiang, Tan Wei, Analysis and Study on the Application of 4G Technology to Power Quality Monitoring [J]. Electrical Measurement and Instruments, 2015, No. 6, 44-48.

[6] Paul A. Garris, Robert Ensman, John Poehlman, etc. Wireless transmission of fast-scan cyclic voltammetry at a carbon-fiber[J] proof of principle. Journal of Neuroscience Methods, 2004, (140), 103-115.

[7] Florian Luisier, Cedric Vonesch, Thierry Blu, etc. Fast interscale wavelet denoising of Poisson-corrupted images[J]. Signal Processing, 2009, (90),415-427.

[8] IEEE Recommended Practice for Monitoring Electric Power Quality,IEEE Std . 2001 . 1159-1995.

[9] Melhorn C J,Davis T D . Bean G E . Voltage Sags : Their Impact on the Utility and Industrial Customers[J],IEEE Transactions on Industry Applications,2002,34(3),549—558. 